\title{
IMPACTO DO PROGRAMA DE EDUCAÇÃO TUTORIAL (PET/MEC) SOBRE A SAÚDE BUCAL EM MUNICÍPIOS DO ESTADO DA BAHIA
}

\author{
Elisabeth Martinez Fonseca ${ }^{\alpha}$ \\ Thamiles Pinheiro Oliveira Araújo ${ }^{a}$ \\ Marina Cartibani ${ }^{a}$ \\ Camila Nobre ${ }^{a}$ \\ Monah Sampaio ${ }^{a}$ \\ Alena Ribeiro Alves Peixoto Medrado ${ }^{b}$
}

\begin{abstract}
Resumo
O presente artigo descreve uma atividade de ensino e extensão desenvolvida por bolsistas integrantes do Programa de Educação Tutorial do curso de odontologia da Escola Bahiana de Medicina e Saúde Pública intitulada "PET sobre os trilhos". Objetiva relatar duas experiências bem sucedidas no âmbito da promoção de saúde bucal em cidades do interior do estado da Bahia. Foram realizadas atividades lúdico-educativas em comunidades dos municípios de São Domingos e Ribeira do Pombal, a exemplo de escovação bucal supervisionada, palestras educativas, jogos e brincadeiras de caráter educativo e distribuição de escovas e cremes dentais. Houve plena participação de bolsistas do grupo PET, com supervisão da tutora. Temáticas relacionadas à promoção de saúde bucal e sistêmica foram abordadas com indivíduos de diferentes faixas etárias. Desta maneira, a relevância deste projeto é atribuída aos impactos sociais no desenvolvimento de conhecimentos e atitudes sobre a adequada manutenção da saúde bucal por parte das comunidades envolvidas, bem como a compreensão acerca das causas e consequências de uma má higiene oral.

Palavras-Chave: Educação em saúde bucal. Saúde Coletiva. Proteção social em saúde. Extensão comunitária.
\end{abstract}

\footnotetext{
Autor correspondente: emf747@gmail.com
}

a. Bolsistas do Programa de Educação Tutorial (PET-ODONTO) do Curso de Odontologia da Escola Bahiana de Medicina e Saúde Pública.

b. Tutora do Programa de Educação Tutorial (PET-ODONTO) e Professora Adjunto do Curso de Odontologia da Escola Bahiana de Medicina e Saúde Pública. alenamedrado@hotmail.com 


\title{
IMPACT OF TUTORIAL EDUCATIONAL PROGRAM (PET / MEC) ON ORAL HEALTH IN CITIES OF BAHIA STATE
}

\begin{abstract}
This article describes an activity of teaching and extension developed by members of the Fellows Program Education Tutorial dentistry course of Bahiana School of Medicine and Public Health, entitled " PET on the rails". Aims to report two successful experiences in promoting oral health in several cities in the state of Bahia. Activities were recreational and educational communities in the municipalities of São Domingos and Ribeira do Pombal, like brushing supervised oral, educational lectures, games and jokes of the educational distribution of toothbrushes and toothpastes. There was full participation of students from the PET group, under the supervision of a teacher. Issues related to the promotion of oral health and systemic been showed with individuals of different age groups. An this, the relevance of this project is attributed to the social impacts on the development of knowledge and attitudes about the proper maintenance of oral health for the communities involved, as well as the understanding of the causes and consequences of poor oral hygiene.

Keywords: Oral health education. Public Health. Social protection in health. Community-Institutional Relations.
\end{abstract}

\section{INTRODUÇÃO}

O Programa de Educação Tutorial (PET) foi criado pelo Ministério da Educação e Cultura (MEC) com o objetivo de apoiar atividades acadêmicas que integram ensino, pesquisa e extensão.(1) Para promover estas atividades, formam-se grupos tutoriais de aprendizagem com alunos de graduação sob a orientação de um tutor-professor, os quais elaboram e executam ações extracurriculares afim de complementar a formação acadêmica dos participantes, atender às necessidades do curso de graduação e promover impacto social nas comunidades que possam ser contempladas pelas atividades do programa.
Um dos maiores desafios encontrados pelos integrantes do ProgramadeEducaçãoTutorial (PET/MEC) é promover impacto social relevante e positivo nas comunidades com as quais os trabalhos são realizados. Em função disto, é preciso sempre buscar conhecer as necessidades da população com que se quer trabalhar, bem como quais as melhores formas de abordagem e possíveis parcerias entre os representantes comunitários, governamentais e institucionais.

Existe grande carência de informações específicas sobre a qualidade da saúde bucal em alguns municípios do Estado da Bahia e há necessidade de realizar 
atividades de Promoção de Saúde Bucal em muitas destas cidades. O apoio dos poderes públicos dos municípios que recebem os estudantes do Programa de Educação Tutorial é imprescidível para a realizaçãode ações de carater educativo e preventivo.

O municipio de São Domingos, localizado na microrregião de Serrinha, (3) apresenta uma população mista, urbana e rural, além de comunidade quilombola. E, o município de Ribeira do Pombal - Bahia, pertence à microrregião de Ribeira do Pombal, (3) fazendo divisa com o Estado de Sergipe. Sendo assim, em virtude do apoio dos municipios de São Domingos e Ribeira do Pombal, o grupo de estudantes integrantes do Programa de Educação Tutorial da Escola Bahiana de Medicina e Saúde Pública visitaram essas cidades e realizaram atividades de educação e promoção de saúde com suas comunidades.

\section{OBJETIVO}

O objetivo deste trabalho é relatar duas experiências bem sucedidas de educação em saúde bucal desenvolvidas pelo grupo PET Odonto Bahiana, nos municípios de São Domingos e Ribeira do Pombal, durante os anos de 2011 e 2012.

\section{MATERIAIS E MÉTODOS}

Os representantes dos municípios contemplados foram contatados por meio de um oficio à prefeitura, o qual descreveu as atividades a serem realizadas, os gastos de manutenção e solicitando acesso às instalações públicas tais como escolas e postos de saúde, bem como o acompanhamento dos respectivos representantes locais (Figura 1).
O grupo atuante foi composto por doze estudantes e integrantes do PET/MEC, do curso de Odontologia da Escola Bahiana de Medicina e Saúde Pública, além de profissionais participantes do Programa de Saúde da Família dos municipios abordados.

As atividades foram realizadas de acordo com a viabilidade das amostras. Foram escolhidos grupos populacionais com base nos critérios de referência do Ministério da Saúde, SBB Brasil 2010:(4) crianças e adolescentes com faixa etária de 05, 12 e 15 até 19, participaram do projeto com intuito de estimular o auto-cuidado com ênfase na saúde bucal através de promoção de saúde.

Os dois municípios selecionados foram contemplados com atividades de orientação e palestras de higiene bucal, as quais foram adaptadas para as necessidades e hábitos culturais previamente pesquisados junto às prefeituras. As atividades foram realizadas em creches e escolas estaduais e municipais, além de feiras de saúde previamente existentes. Sendo assim, o público assistido no geral foi de crianças, adolescentes, adultos e idosos.

O gerenciamento das atividades dependeu diretamente da infra-estrutura dos locais de trabalho e os kits odontológicos distribuidos eram compostos por dentifrício odontológico, escova de dentes e folheto informativos sobre técnicas de escovação (Figura 3). Considerando a realidade dos recursos de saúde de cada população, foram feitas interferências através de palestras, dinâmicas, jogos e atividades educativas em saúde bucal e geral (Figura 2). Os estudantes envolvidos prepararam situações que poderiam ser trabalhadas em coletivo e individualmente. Desta forma, todos os participantes das feiras de saúde, escolas e creches foram acolhidos e tiveram suas dúvidas sanadas (Figura 4). 


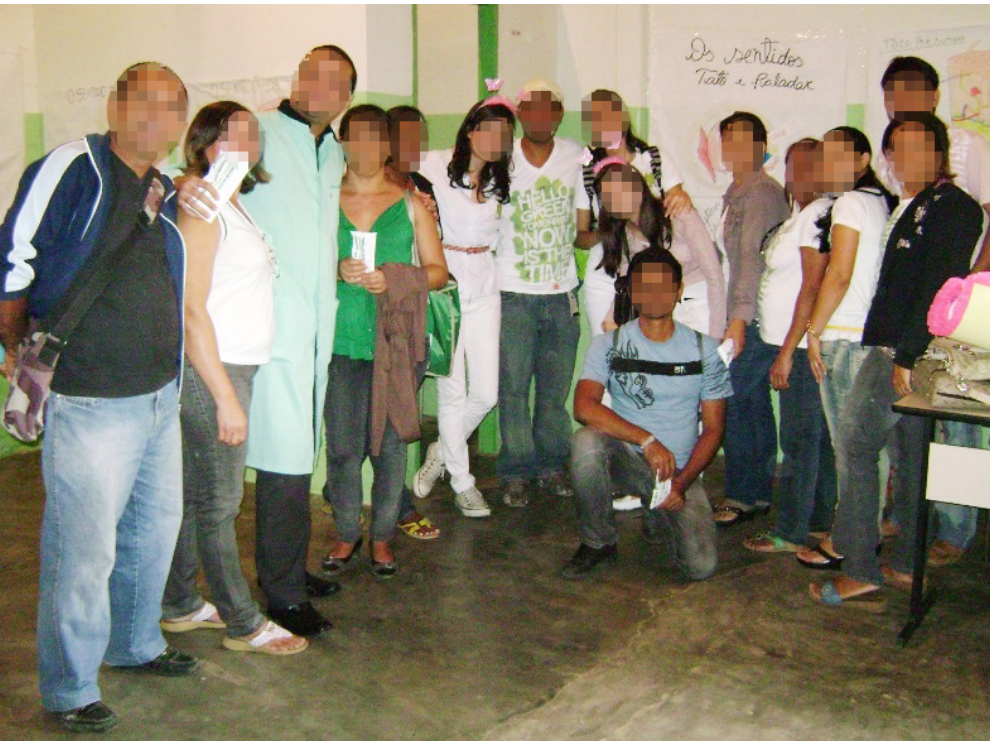

Figura 1. Integração de acadêmicos, agentes comunitários, professores da rede pública e dentistas do PSF em escola pública do Município de Ribeira do Pombal

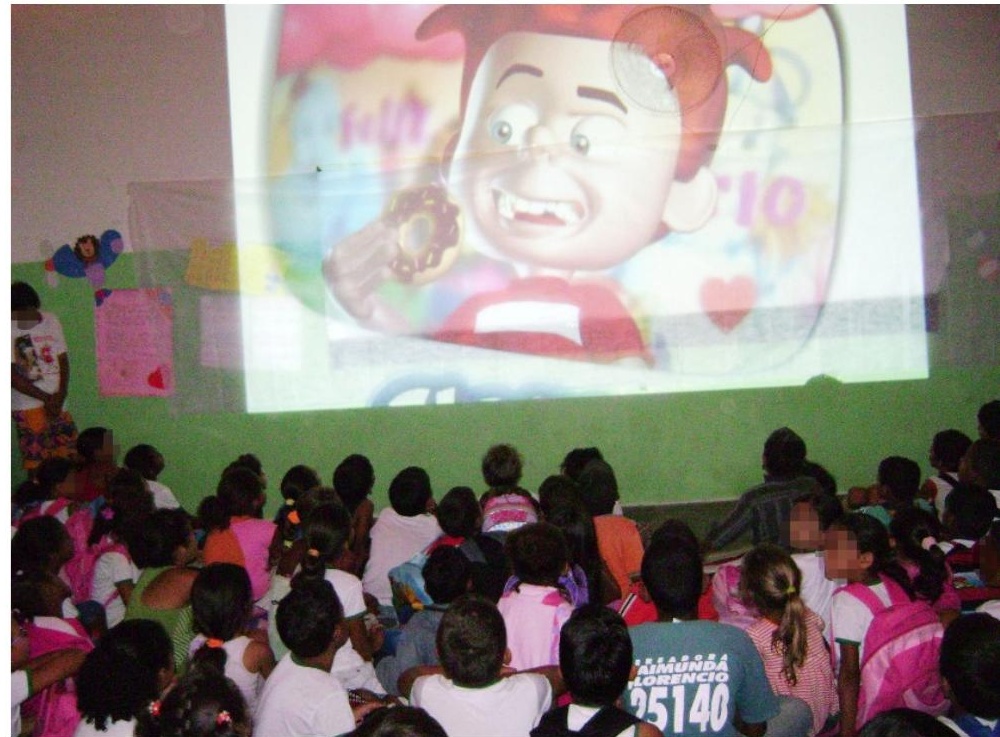

Figura 2. Exibição de filme sobre Saúde bucal para jovens e crianças de escolas públicas

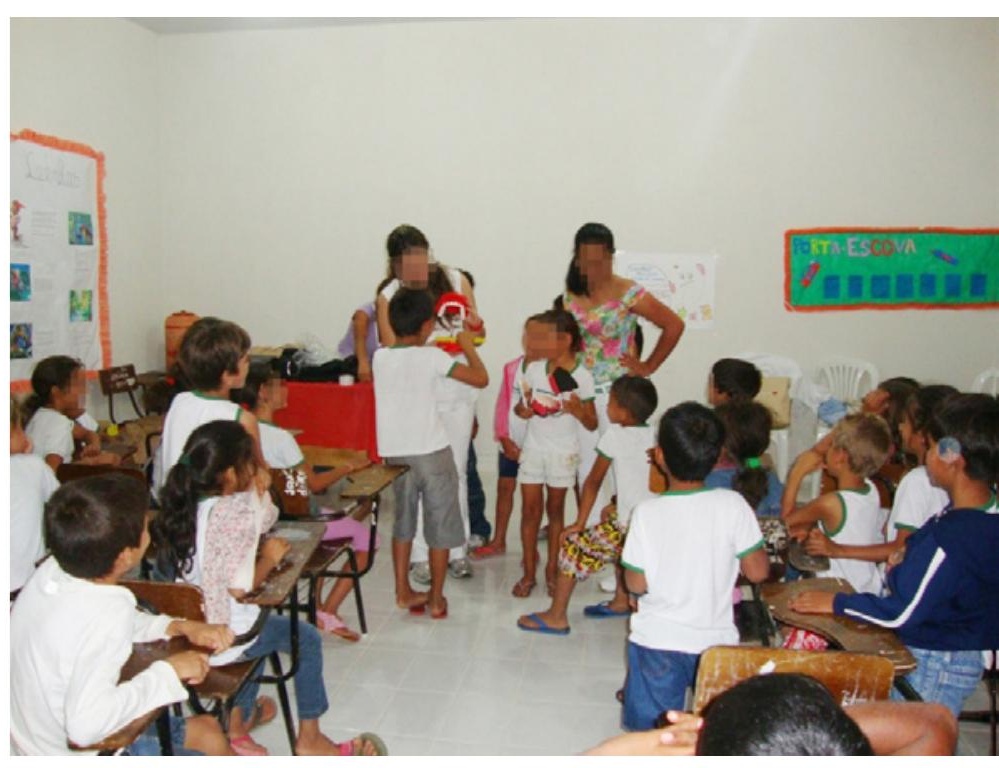

Figura 4. Atividade lúdica sobre saúde bucal em sala de aula

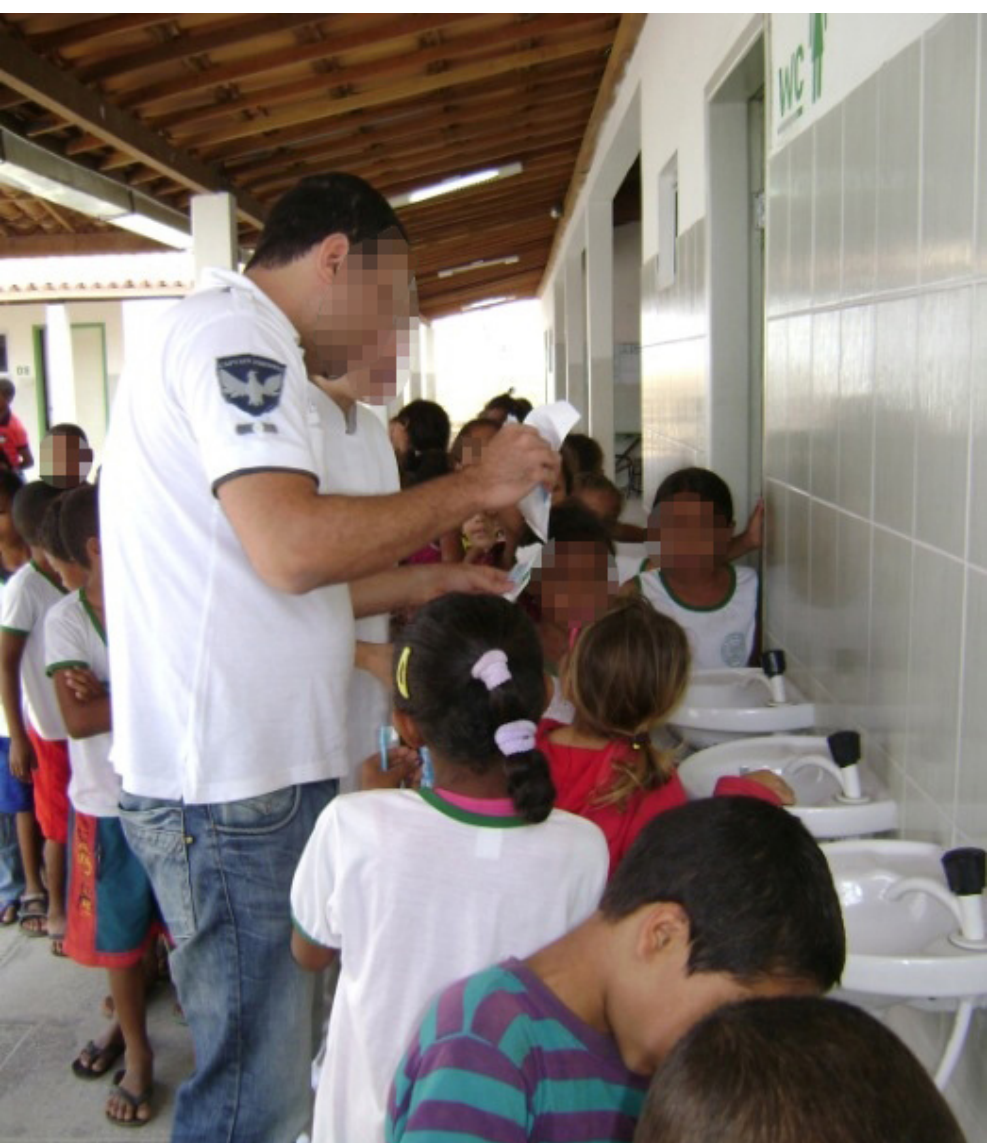

Figura 3. Escovação Supervisionada 


\section{RESULTADOS}

Todas as atividades foram realizadas em escolas e creches públicas dos municipios de São domingos, Ribeira do pombal e adjacências, contemplando um público de aproximadamente 3.000 crianças, adolescentes e jovens adultos em cada municipio. Houve também a capacitação de professores da rede pública para demonstração ao longo do ano letivo sobre a importância da utilização de fio dental e técnicas de escovação.

Antes de dar inicio a escovação supervisionada, exame de mucosa oral, unidades dentárias e aplicação de flúor dental, os jovens eram encaminhados a conferências com alunos do PET odonto Bahiana sobre saúde bucal e higiene pessoal.

Os alunos e crianças das creches eram divididos de acordo com ano letivo e faixa etária, contendo aproximadamente 50 pessoas por grupo. Eles aguardavam em sala de aula pela conferência dos alunos do programa, cujo os temas tratados estavam sempre relacionados à hábitos de higiene pessoal e íntima, cronologia da erupção dentária, cariologia, tecnicas de escovação, doenças gengivais e periodontais, comprometimentos pulpares, doenças sistêmicas com manifestações bucais e vice versa. Contudo, os discussos eram adaptados e estudados de acordo com a faixa etária e grau de escolaridade de cada grupo, afim de promover um maior entendimento dos temas abordados.

Após as conferências, os alunos do PET incentivavam os jovens a questionarem o que foi discutido e a participarem de atividades de carater lúdico-educativo sobre os conhecimentos aprendidos em sala. Sendo realizados jogos como "memória", "quebra-cabeças", "caça-palavras", teatro de fantoches e questionamentos sempre sobre os assuntos aprendidos. Os alunos do Programa não participavam das brincadeiras, apenas supervisionavam e incentivavam o inicio do momento de descontração e aplicação do que foi aprendido, entendendo que esta aplicação do conhecimento com atividades lúdicas é tão importante quanto a própria conferência ministrada.
Após a realização das atividades sobre conhecimentos de saúde, os jovens participantes eram encaminhados a uma área pré-idealizada para a realização de escovação supervisionada com os kit odontológicos doados pelas prefeituras. Os alunos do Programa de Educação Tutorial orientaram que os kits fossem mantidos da unidade pública de ensino, visto que os alunos passavam praticamente todo o dia nas escolas e creches, o que os incentivaria a manter o hábito de auto-cuidado.

O grupo de alunos e colaboradores do Programa de Educação Tutorial da Escola Bahiana de Medicina e Saúde Pública sempre foi bem recebido em cada comunidade visitada, contando com a participação efetiva dos líderes, representantes e agentes comunitários, além dos profissionais capacitados que trabalham no Programa de Saúde da Família (PSF) das regiões onde as atividades estavam sendo realizadas. Após e durante as atividades. o grupo teve, inclusive, repercussão na mídia das regiões onde visitaram, aparecendo em jornais comunitários e rádios locais.

\section{DISCUSSÃO}

A construção do conhecimento em saúde bucal é um processo que ocorre de diversas formas. Os jovens aprendem autocuidados e higiene pessoal através da convivência familiar e pedagógica escolar, podendo absorver de forma ampla ou limitada tais conhecimentos, dependendo de qual modo eles são abordados e quais os valores sociais individualizados dessa carga educacional.

Segundo Figueira e Leite, ${ }^{(5)}$ atualmente, as atividades desenvolvidas pelas técnicas de higiene dental nas escolas se concentram basicamente na realização de escovação supervisionada e aplicação tópica de flúor gel. As atividades educativas, em função do alto número de escolas a visitar $\mathrm{e}$ poucos recursos humanos, ficam em segundo plano. Esta situação é ainda mais agravada quando 
observa-se que muitas prefeituras cobram metas de procedimentos coletivos. Sendo assim, o período de tempo alargado entre uma atividade educativa e outra, quando não atinge a população, gera o esquecimento e se torna, geralmente, ineficaz.

É importante observar que as atividades lúdicas facilitam o processo de aprendizado. Através de interações harmônicas com brincadeiras, conhecimentos e jogos, as crianças administram melhor os conhecimentos que foram explanados em sala de aula e podem levá-lo para a maior compreensão do mundo em que vivem, quais papeis sociais elas e suas famílias ocupam, quais os conhecimentos podem ser empregados no dia a dia e afetividade, por estimular a interação com outras crianças durante essas atividades. Dessa forma, acredita-se que os conceitos de autocuidado e saúde bucal expostos nas conferências podem ser mais aprendidos com o estímulo a questionamentos e brincadeiras entre os grupos.

De acordo com Alves et al.,(6) dentre os modelos de educação em saúde, o modelo dialógico favorece o reconhecimento dos usuários enquanto sujeitos portadores de saberes sobre o processo saúde-doença-cuidado e de suas próprias condições de vida. Neste mesmo âmbito, o modelo contribui para a compreensão mais ampla das necessidades de saúde dos sujeitos com a humanização da ação educativa, tornando o processo mais sensível a seus destinatários quando estimula a troca dos conhecimentos aprendidos.

Para que as atividades de saúde sejam duradouras e efetivas, é preferível que haja uma parceria entre os ciclos sociais presentes na vida das crianças e jovens e estes ciclos devem ser estimulados a tratar de assuntos semelhantes e contundentes. Ou seja, a família, escola, os pais e a própria comunidade em que essas pessoas vivem, devem ser motivadas a tratar com mais clareza e compreensão assuntos que se relacionem as suas próprias condições de vida. Portanto, para que uma atividade de saúde, por exemplo, tenha discurssos mais efetivos, é preciso que os agentes conheçam os núcleos envolvidos e se adaptem para se aproximar cada vez mais de seu grupo de ação.
Segundo Franchin et al.,(7) a promoção de saúde bucal pode e deve se realizar para além dos limites do consultório odontológico. $\mathrm{E}$, os professores são considerados agentes multiplicadores de informações e hábitos de saúde bucal, quando são efetivamente capacitados para essas situações. Os professores são extremamente importantes para a manutenção deste tipo de conhecimento em sala de aula, uma vez que são profissionais com conhecimento abrangente sobre técnicas metodológicas, relações interpessoais e já apresentam certo envolvimento emocional e psicológico com os alunos do seu grupo.

Em função disso, quando os professores estimulam atividades lúdico-explanatórias em grupos nas suas escolas, eles favorecem o aprendizado do que está sendo discutido, uma vez que as crianças se envolvem em um meio coletivo, social e intelectual, onde as ações trabalhadas se complementam e se desenvolvem amplamente.

Portanto, para as atividades de saúde bucal exerçam um impacto positivo na comunidade, é preciso a integração de diversas áreas de conhecimento e, também, diferentes formas de explanação desses conhecimentos. Sendo assim, os grupos de trabalho formados devem se adequar a demanda e exercer manejo e controle sobre o gerenciamento das atividades. A organização das conferências concomitantemente as atividades lúdicas é essencial para a prática do conhecimento e potêncial para promover impacto positivo na multiplicação e efetividade de conhecimentos em saúde bucal nas populações em que são trabalhadas.

\section{CONCLUSÃO}

A parceria dos alunos do Programa de Educação Tutorial do curso de odontologia da Escola Bahiana de Medicina e Saúde Pública com as escolas, comunidades, famílias e poderes públicos buscou ampliar a divulgação dos conhecimentos que já são costumeiramente transmitidos à população nas comunidades em que foram realizadas as atividades de promoção de saúde. Com esta atividade observou-se que avaliar as estratégias de promoção 
de saúde bucal é um desafio que requer conhecimentos técnicos, administrativos e teóricos.

Portanto, contar com a participação dos diferentes núcleos comunitários que estão diretamente envolvidos com as populações trabalhadas faz com que estas estratégias sejam potencializadas e complementadas. Estas ações permitem a construção de referenciais e valores que apoiem os processos de fortalecimento e mudanças nas estratégias de educação em saúde bucal locais, fazendo com que parte da carência de informações específicas sejam tratadas.

Em contra partida, existe a necessidade de retornar a estes centros municipais para retomar o trabalho e abservar se as atividades que foram desenvolvidas e repassadas dos alunos do programa para os professores das escolas e creches estão sendo efetivadas. Apesar dos crescentes estudos na área de promoção de saúde bucal no Brasil, ainda não é comum observar trabalhos de retomadas às comunidades para re-afirmação das instruções e conhecimentos em saúde.

\section{REFERÊNCIAS}

1. Iguatemy ML. Educação tutorial no ensino presencial: uma analise sobre o PET. In: Brasil. Ministério da Educação. PET - Programa de Educação Tutorial: estratégia para o desenvolvimento da graduação. Brasilia, DF: Ministério da Educação, 2007. p. 12-21.

2. Giuseppe RA. Epidemiologia e saúde bucal coletiva: um caminhar compartilhado. Ciênc. saúde coletiva [serial on the Internet]. 2006. [aceso em 10 out 2013]; 11(1):105-114. Disponível em: http://dx.doi. org/10.1590/S1413-81232006000100018.

3. Instituto Brasileiro de Geografia e Estatística [homepage na internet]. População, Contagem. [acesso em 10 out 2O13]. Disponivel em: http://www.ibge.gov.br

4. Projeto SBB Brasil, 2010 [homepage na internet]. Materiais e métodos para pesquisas. [Acesso em 10 out 2013]. Disponível em: http://dab.saude.gov.br/ cnsb/sbbrasil/index.html

5. Figueira TR, Leite, ICG. Percepções, conhecimentos e práticas em saúde bucal de escolares. RGO. 2008;56(1): 27-32.
6. Alves VS. Um modelo de educação em saúde para o Programa Saúde da Família: pela integralidade da atenção e reorientação do modelo assistencial. Interface (Botucatu) [serial on the Internet]. 2005. [Acesso em 10 out 2013]; 9(16):3952. Disponível em: http://dx.doi.org/10.159O/S141432832005000100004.

7. Franchin V, Basting BT, et al. A importância do professor como agente multiplicador de saúde bucal. Rev ABENO. 2006;6(2):102-108.

\section{Franco TB, Merhy EE. Programa de Saúde} da Família (PSF): contradições de um programa destinado à mudança do modelo tecnoassistencial In: Merhy EE, Magalhães Júnior HM, Rimoli J, Franco TB, Bueno WS. O trabalho em saúde: olhando e experienciando o SUS no cotidiano. 3.ed. São Paulo: Hucitec; 2006. p. 53-124.

9. Denti I A. Programa de saúde da família: suas possibilidades e limites na promoção da saúde e do trabalho com a família. Texto \& contexto enferm. 2000; 9(2):699-713.

10. Giudice ACMP, Pezzato LM, Botazzo C. Evaluation practices: reflections on the integration of oral health in the Family Health Team. Saúde debate [serial on the Internet]. 2013 [acessado em 10 out 2013];37(96):32-42. Disponível em: http:// dx.doi.org/10.1590/S0103-11042013000100005.

11.Costa GMC et al. Promoção de saúde nas escolas na perspectiva de professores do ensino fundamental. Rev. Eletr. Enf. 2013;15(O2):506-15

12. Giudice ACMP, Pezzato LM, Botazzo C. Práticas avaliativas: reflexões acerca da inserção da saúde bucal na Equipe de Saúde da Família. Saúde Debate. 2013;37(96): 32-42.

13. Kusma SZ, Moysés ST, Moysés SJ. Promoção da saúde: perspectivas avaliativas para a saúde bucal na atenção primária em saúde Health promotion: perspectives for evaluation of oral health in primary healthcare. Cad. saúde pública. 2012;28:9-19.

14. Rodrigues $A A A O$ et al. Interaction between education, services, and the community: the experience of a PET-Saúde Project. Revista Brasileira de Educação Médica. 2012;36(1):184-192.

15. Bulgareli JV, Faria ET et al. Informações da atenção secundária em Odontologia para avaliação dos modelos de atenção à saúde. Rev. odontol. UNESP. 2013;42(4): 229-236. 